\title{
Im Olymp der Medizinzeitschriften
}

Ein medizinisches Fachjournal kann vieles sein: ein Organ einer medizinischen Fachgesellschaft, ein Informationsmedium für Anwender oder ein Forum für die Publikation von Forschungsergebnissen. Publikation aber bedeutet Öffentlichmachung, und die gelingt nur so gut, wie gut das Forum selbst öffentlich zugänglich ist. Für die öffentliche Beachtung einer Erkenntnis ist daher die Reichweite ihres Trägermediums nicht minder bedeutend als der Inhalt selbst.

Noch vor 10 Jahren waren die aktuellen wissenschaftlichen Fachinformationen im wesentlichen auf öffentliche Bibliotheken beschränkt. Wer sich kundig machen wollte über das, was noch zu neu war, um Eingang in ein Lehrbuch zu finden, war auf diese Institutionen angewiesen. Es sei denn, man abonnierte das eine oder andere Journal selbst. Dieses Vorgehen dürfte allerdings im Hinblick auf eine umfassende Information für die meisten Forscher viel zu aufwendig sein.

Seit uns mit der Einführung der CD-Rom Speicherkapazitäten zur Verfügung stehen, die auch grosse Datenmengen handhabbar und mobil machen, hat sich in der Medizin viel geändert. Medline, der Bestandskatalog der American National Library of Medicine mit seinen etwa 3800 Journalen im laufenden Bestand, wurde weltweit zur Informationsquelle Nummer eins. Sie ist inzwischen über das Internet für jeden zu Hause zugänglich. Die breite Abdeckung mit neuesten Forschungsdaten aus der Medizin auf diesem Informationsweg hat aber dazu geführt, dass sich Gesundheitspolitiker, Forscher und Anwender nur noch ausnahmsweise der Mühe unterziehen, über diese Quelle hinaus Publikationen zu verfolgen. Medline ist so zum Massstab geworden. Das muss man nicht uneingeschränkt gut finden, trotzdem kann an dieser Tatsache keiner vorbeikommen.

Deshalb war es auch ein vorrangiges Bestreben der FORSCHENDEN KOMPLEMENTÄRMEDIZIN, von Anfang an in diesen Olymp der medizinischen Fachzeitschriften aufgenommen zu werden. Was nur wenigen vergönnt ist, hat unsere Zeitschrift nun geschafft: Sie ist gelistet in Medline. Parallel zur Medline-Listung erfolgt auch die Aufnahme in Current Contents, eine weitere amerikanische Datenbank mit einer grossen Reichweite, die sich besonders um Aktualität bemüht. Wöchentlich erhalten die Abonnenten von
Current Contents eine neue CD-Rom, die sämtliche Zusammenfassungen aller gelisteten Journale, die in der Woche neu erschienen sind, enthält. Nachrichtenagenturen und Redaktionsteams grosser Zeitungen bedienen sich auf der Suche nach interessanten Neuigkeiten routinemässig dieser Datenbank.

Mit der Listung in Medline und Current Contents wird der Anreiz für Autoren, qualitativ hochwertige Arbeiten einzureichen, massiv erhöht - zum Vorteil der Leser. Die Schriftleitung der FORSCHENDEN KOMPLEMENTÄRMEDIZIN ist um so mehr bemüht, den Review-Prozess weiter zu optimieren und den Autoren nicht nur kompetente, faire und sachlich konstruktive Begutachtungen zukommen zu lassen, sondern auch ein zügiges Procedere zu garantieren. Ausserdem möchten wir alle in Frage kommenden Arbeitsgruppen ermuntern, uns auch ihre gut dokumentierten Studien mit «negativem» Ausgang zuzuschicken. Im Interesse der Patienten und der Forschung ist auch eine umfassende Information über die Grenzen der Wirksamkeit und über unerwünschte Nebenwirkungen von Therapien ein essentielles Anliegen dieser Zeitschrift.

Die grossen Fortschritte in der Dokumentation, Beschaffung und Auswertung wissenschaftlicher Arbeiten bergen jedoch auch Risiken in sich. Vor allem die Gefahr einer einseitigen Bewertung diagnostischer und therapeutischer Massnahmen ist hierbei zu nennen. Einiges deutet darauf hin, dass die weltweite Kommunikation und die durch sie ausgelöste Flut wissenschaftlicher Daten zu einer gewissen Gleichschaltung in der Wissenschaft geführt haben, die nicht nur positive Aspekte im Sinne einer qualitativen Standardisierung besitzt. So wurde beispielsweise mit der in letzter Zeit immer beliebteren Methode der Metaanalyse eine Hierarchie von Qualitätskriterien entwickelt, die den klinischen Alltag nur unzureichend widerspiegelt. Da über solche Probleme stärker diskutiert werden muss, wollen wir uns noch intensiver darum bemühen, neben wissenschaftlichen Originalarbeiten auch methodologische Arbeiten zu publizieren und neben den biologischen auch geisteswissenschaftliche Argumentationsweisen zu Wort kommen zu lassen.

Die Aufnahme unserer Zeitschrift in Medline und Current Contents freut uns auch aus einem anderen Grund: Die Schriftleiter engagieren sich - jeder mit seinen Prioritäten - bereits seit langem

\section{KARGER}

Fax +49761452071

E-mail kargergmbh@aol.com

www.karger.com (c) 1999 S. Karger GmbH, Freiburg

Accessible online at:

http://BioMedNet.com/karger 
für diese Form von Medizin. Leider fand dieser Bereich, der für die Zukunft ein immenses Potential besitzt, im grossen Chor der medizinischen Forschung bisher nur wenig Beachtung. Mit der Würdigung einer Zeitschrift wie der FORSCHENDEN KOMPLEMENTÄRMEDIZIN ist aber auch die Würdigung dieses Medizinbereiches verbunden.

Vielleicht hängen die Probleme auch damit zusammen, dass darüber, was «Komplementärmedizin» wirklich bedeutet und in welchem Zusammenhang sie mit der mitteleuropäischen klassischen Naturheilkunde steht, bisher alles andere als Einigkeit herrscht. Nachdem die klassische Naturheilkunde in unserer Zeitschrift immer stärker an Gewicht gewann - was nicht zuletzt durch die Zusammenarbeit mit der Europäischen Gesellschaft für klassische
Naturheilkunde und mit der Schweizerischen Medizinischen Gesellschaft für Phytotherapie dokumentiert wurde -, haben wir daher ernsthaft darüber diskutiert, inwieweit dies nicht auch im Titel der Zeitschrift zum Ausdruck kommen müsste. Denn als komplementär empfinden sich die meisten Vertreter der klassischen Naturheilverfahren nicht. Da diese Diskussion noch im Gange ist, möchten wir unsere Leser herzlich dazu aufrufen, sich an diesem Prozess zu beteiligen. Wissenschaft muss flexibel sein, sie darf aber die Grenzen, innerhalb deren sie agiert, nicht aus den Augen verlieren. Helfen Sie uns für unser Anliegen einen gemeinsamen Nenner zu finden!

Die Schriftleitung 